\title{
Modulation of the dephasing time for a magnetoplasma in a quantum well
}

\author{
M. W. Wu and H. Haug \\ Institut für Theoretische Physik, J.W. Goethe Universität Frankfurt, Robert-Mayer-Straße 8, D-60054 Frankfurt a. M., \\ Germany \\ (October 15, 2018; E-mail: wu@mandala.th.physik.uni-frankfurt.de)
}

\begin{abstract}
We investigate the femtosecond kinetics of optically excited 2D magneto-plasma. We calculate the femtosecond dephasing and relaxation kinetics of the laser pulse excited magneto-plasma due to bare Coulomb potential scattering, because screening is under these conditions of minor importance. By taking into account four Landau subbands in both the conduction band and the valence band, we are now able to extend our earlier study [Phys. Rev. B 58, 1998, in print] to lower magnetic fields. We can also fix the magnetic field and change the detuning to further investigate the carrier density-dependence of the dephasing time. For both cases, we predict strong modulation in the dephasing time.
\end{abstract}

Keywords: A. quantum wells, A. semiconductors, D. electron-electron interactions, D. optical properties

Numerous experimental and theoretical studies have been devoted to the problem of transient charge fluctuations induced by femtosecond pulse excitation in semiconductors which can be studied through nonlinear-optical effects to elucidate many-body phenomena, such as time-dependent Coulomb correlations. Most of the experimental studies have been performed without magnetic field [1] 3]. The few femtosecond optical studies in the presence of a strong magnetic field focused on low-density magneto-excitons [4] 7]. With strong resonant laser pulses which excite a dense carrier system — with a density above the Mott ionization density - in a strong magnetic field, one can study the relaxation and dephasing kinetics of a magnetoplasma. This problem becomes important as experimental studies of the relaxation and dephasing kinetics in QW's and superlattices are in progress 8 .

Recently, we presented a first kinetic study for a femtosecond laser-pulse excited 2D dense non-equilibrium magnetoplasma in a QW in the framework of the semiconductor Bloch equations combined with Coulomb scattering rates [10]. We assumed an additional weak lateral confinement which lifts the degeneracy of the Landau levels partially. We expanded the density matrix of a two-band (i.e., the conduction band and the valence band) semiconductor in the eigenfunctions of the $2 \mathrm{D}$ electron in the presence of the strong magnetic field and the weak parabolic confinement. We formulated the scattering terms for the population distribution functions of the various Landau subbands and for the optically induced polarization components between the Landau-subbands in the conduction and valence band in the form of non-Markovian quantum kinetic scattering integrals 3 and in the form of semiclassical Boltzmann-type scattering rates. We calculated the time-resolved (TR) and time-integrated (TI) four-wave mixing (FWM) signals for two 50 fs pulses by taking into account up to three Landau subbands in both the valance band and the conduction band. The carrier frequency of the two delayed pulses is tuned slightly above the unrenormalized energy gap. We simplified the problem by assuming equal effective electron and hole masses, as it can be approximately realized in strained QW's. Naturally, unequal effective masses will lead to more complicated quantum beat structures in the FWM signals and modify to some extent also the resulting relaxation and dephasing rates. Thus our studies should be seen only as an idealized model calculations. Bare Coulomb potential is used in our calculation because screening is under these strong confinement conditions of minor importance (see e.g. Ref. [9]). Naturally in the limit of vanishing magnetic field a Boltzmann kinetics with a bare Coulomb potential is not justified.

We find in our preceding paper [10] that the FWM signals exhibit quantum beats mainly with twice the cyclotron frequency. Contrary to general expectations, we find no pronounced slowing down of the dephasing with increasing magnetic field. On the contrary, one obtains in same ranges of the magnetic field a decreasing dephasing time because of the increase of the Coulomb matrix elements and the number of states in a given Landau subband. In the situation when the loss of scattering channels exceeds these increasing effects, one gets a slight increase of the dephasing time. However, details of the strongly modulated scattering kinetics depend sensitively on the detuning, the plasma density, and the spectral pulse width relative to the cyclotron frequency.

As discussed in our previous paper, we took only three Landau subbands in our calculation. This is mainly because of the expansion of number of matrix elements of Coulomb scattering increases as $N^{4}$ with $N$ being the total number of Landau subbands considered. With $N=3$ in our previous calculation, the number of form factors is already 81. However, such low number of Landau subbands limits us to the magnetic fields higher than 10 T. More Landau subbands are necessary in order to extend the kinetics to lower magnetic fields or for larger detunings.

In this report, we take into account four Landau subbands in both the conduction band and the valence band. 256 matrix elements of Coulomb scattering are calculated in the same way as discussed in the Appendix of Ref. [10]. So 
we can investigate the femtosecond dephasing and relaxation kinetics of magnetoplasma with magnetic field $B>6 \mathrm{~T}$. We can also fix the magnetic field and tune the laser pulses over a few Landau subband transitions. We find strong modulation of the dephasing time both for variations of the magnetic field and the detuning. These modulations could not be seen fully in our previous paper due to the small range of available $B$ fields limited by three considered Landau subbands.

The semiconductor Bloch equations are all the same as those in our previous paper [10]:

$$
\dot{\rho}_{\nu, n, \nu^{\prime}, n^{\prime}, k}=\left.\dot{\rho}_{\nu, n, \nu^{\prime}, n^{\prime}, k}\right|_{\mathrm{coh}}+\left.\dot{\rho}_{\nu, n, \nu^{\prime}, n^{\prime}, k}\right|_{\text {scatt }},
$$

with $\rho_{\nu, n, \nu^{\prime}, n^{\prime}, k}$ representing the single-particle density matrix with the band indices $\left\{\nu, \nu^{\prime}\right\}=\{c, v\}$ and the correspondung Landau subbands $\left\{n, n^{\prime}\right\}$. The diagonal elements describe the carrier distribution functions $\rho_{\nu, n, \nu, n, k}=f_{\nu n k}$ of the $n$-th Landau subband and the wavevector $k$, and the off-diagonal elements describe the interband polarization components, e.g. $\rho_{c, n, v, n, k}=P_{n k} e^{-i \omega t}$. For the assumed e-h symmetry, $f_{e n k} \equiv f_{h n k} \equiv f_{n k}$ and the polarization has only components between subbands of the same quantum number $\mathrm{n}$ in the conduction and valence band, which simplifies the problem considerably. The coherent parts of the equations of motion for the distribution functions and the polarization components include Hartree-Fock contributions and can be found in our previous paper. So can the explicit forms of the scattering rates. However in this report, we only take the Markovian limit. The Landau index $n$ in our present study ranges from 0 to 3 .

We use the same material parameters of the quantum well as our previous paper. We perform a numerical study of the Bloch equations in the Boltzmann limit to calculate TR and TI FWM signals in order to study the effective dephasing time. To do so, we use two delayed Gaussian pulses of a width of $50 \mathrm{fs}$ and a variable delay time $\tau$, $E_{0}(t)=E_{0}(t)+E_{0}(t-\tau) e^{i \varphi}$ with the relative phase $\varphi=\left(\mathbf{k}_{2}-\mathbf{k}_{1}\right) \cdot \mathbf{x}$ resulting from the different propagation directions $\mathbf{k}_{1}$ and $\mathbf{k}_{2}$. We use an adiabatic projection technique with respect to this phase in order to calculate the polarization in the FWM direction with wavevector $2 \mathbf{k}_{2}-\mathbf{k}_{1}$ described in detail in Ref. [11]. This technique is suitable for optically thin crystals, where the spatial dependence can be treated adiabatically [12]. The intensity of each pulse is given by $\int_{-\infty}^{\infty} d E_{0}(t) d t=\chi \pi$ with $\chi$ denoting the fraction of a $\pi$-pulse defined without local field corrections and $d$ being the optical-dipole matrix element. Differing from our previous paper where we discussed both the intermediate density case $(\chi=0.1)$ and high density case $(\chi=0.3)$, in this study we focus only on the intermediate density case with $\chi$ fixed to 0.1 .

Our main results are plotted in Figs. 1 and 3. In Fig. 1 we plot the effective dephasing time as function of magnetic field $B$ for pulses with detuning $\Delta_{0}=26.4 \mathrm{meV}$, which is the same value used in our previous calculation [10]. The effective dephasing time $T_{\text {eff }}$ is obtained from the decay of the TI-FWM signal with the delay time $\tau$ written in the form $\propto \exp \left(-\tau / T_{\text {eff }}\right)$. The solid curve is our present calculation with 4 Landau subbands and the dashed curve is our earlier one with 3 Landau subbands. We found they coincide above $B=15 \mathrm{~T}$ which is in agreement with our discussion that 3 Landau subbands are only good for high magnetic fields and one needs to include more Landau subbands for lower magnetic fields. We further find the modulation of dephasing time as it first decreases with decreasing magnetic field and increases again when $B$ decreases from $7 \mathrm{~T}$ to $6 \mathrm{~T}$. We speculate that more modulations occur as the magnetic field is still lower. However, we cannot push our calculation to lower fields because that would require even more Landau subbands.

This modulation can be well understood in the way of our previous discussion [10]. For fixed pulses, several effects compete with each other when the magnetic field increases. On one hand, the number of Landau subbands which contribute to the Coulomb scattering kinetics decreases. In particular the contributions to the dephasing from the intra- and inter-subband scattering of the higher Landau subbands as well as the inter-subband scattering between the higher and lower subbands decrease. For large populations in one subband the Pauli blocking may further reduce also the intra-band scattering rates. All these effects (we refer to them as effects I in the following) increase the dephasing time. On the other hand, with increasing $B$ field the degeneracy of Landau subbands increases and the matrix elements of the Coulomb scattering become larger. Moreover, an increasing degeneracy also increases the scattering rates. Both the increased degeneracy and the increased Coulomb matrix elements (effects II) reduce the dephasing time. When effects I dominate over effects II, one observes increase of the dephasing time. Otherwise, a decrease of the dephasing time results.

In order to further understand the properties of dephasing, we change the detuning for a fixed magnetic field of $B=7$ T. In Fig. 2 we illustrate the pulse spectra tuned at $-20 \mathrm{meV}$ which is far below the band gap (solid curve) and at $40 \mathrm{meV}$ which is deep inside the band (dashed curve). We tune the laser pulses from around $-35 \mathrm{meV}$ to 40 $\mathrm{meV}$ and calculate the effective dephasing time. The resulting dephasing time is plotted in Fig. 3 as function of the detuning $\Delta_{0}$. From Fig. 3 one can see strong modulations of the dephasing time. When the laser pulses are tuned far away from the lowest optical transition, the excitation is very small and the dephasing time is independent on 
the detuning (and the related carrier densities). However the detuning strongly affects the dephasing time when it is larger than 0 . We find the dephasing time reaches minima when the laser pulses are tuned resonantly at $P_{0}$ and $P_{1}$. Another minimum is observed when $\Delta_{0}$ sits between $P_{0}$ and $P_{1}$ and the pulse excites comparable populations in both bands.

For fixed magnetic field, the matrix elements of Coulomb scattering are fixed. The dephasing time is mainly modulated by the occupation of Landau subbands with varying detuning of the laser pulse. When the pulses are resonant with an optical transition, the carriers mainly populate the corresponding Landau subband. This makes the Coulomb scattering more efficient as the scattering rate increases superlinearly with the carrier density. Therefore one observes a minimum in the dephasing time. It is noted here that the distribution function in this calculation is smaller than 0.5 even after the second pulse. This rules out a dominant contribution of Pauli blocking which makes the dephasing times longer as discussed before. The third minimum between $P_{0}$ and $P_{1}$ in Fig. 3 comes from the fact that the center of the pulse sits just between the two lowest optical transitions and it pumps carriers with both of its tails. In this situation the lowest two Landau subbands both get relatively large excitations and therefore lead to the fast dephasing. It is noted that not withstanding the fact that we plotted the detuning to -35 meV, the physically meaningful range is only $\Delta_{0}>0$, where the carrier-carrier scattering dominates over other processes.

In conclusion, we have discovered modulations of the effective dephasing time of $2 \mathrm{D}$ magnetoplasma by either fixing the detuning and changing the magnetic field or fixing the magnetic field and changing the detuning.

We acknowledge financial support by the DFG within the DFG-Schwerpunkt "Quantenkohärenz in Halbleiter". Interesting discussions with D.S. Chemla and H. Roskos are appreciated.

[1] Proceedings of the Third International Workshop on Nonlinear Optics and Excitation Kinetics in Semiconductors, Bad Honnef, Germany [Phys. Stat. Sol. B 173, 1992, 11].

[2] Shah, J., Ultrafast Spectroscopy of Semiconductors and Semiconductor Microstructures (Springer, Berlin, 1996).

[3] Haug, H and Jauho, A.P., Quantum Kinetics in Transport and Optics of Semiconductors (Springer, Berlin, 1996).

[4] Stafford, C., Schmitt-Rink, S. and Schaefer, W., Phys. Rev. B 41, 1990, 10000.

[5] Glutsch, S. and Chemla, D.S., Phys. Rev. B 52, 1995, 8317.

[6] Siegner, U., Bar-Ad, S. and Chemla, D.S., Chem. Phys. 210, 1996, 155.

[7] Rappen, T., Mohs, G. and Wegener, M., Appl. Phys. Lett. 63, 1993, 1222.

[8] Private communications by D.S. Chemla and H. Roskos.

[9] Haug, H. and Koch, S.W., Quantum Theory of the Optical and Electronic Properties of Semiconductors (World Sientific, Singapore, 1994)

[10] Wu, M.W. and Haug, H., Phys. Rev. B 58, 1998, in print

[11] Bányai, L., Reitsamer, E. and Haug, H., J. Opt. Soc. Am. B 13, 1996, 1278.

[12] Lindberg, M., Binder, R. and Koch, S.W., Phys. Rev. A 45, 1996, 1865. 


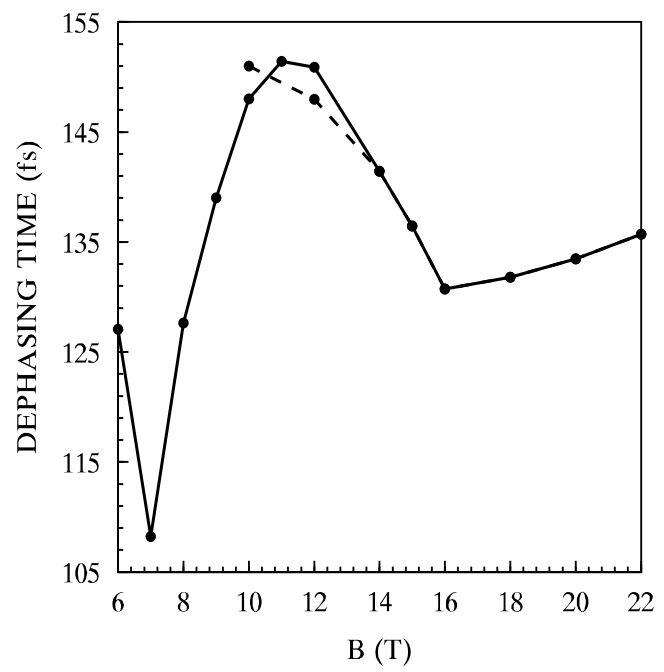

FIG. 1. Dephasing time as a function of $B$ for fixed detuning $\Delta_{0}=26.4 \mathrm{meV}$. The dashed curve gives the result of Ref. 10 calculated with only three pairs of Landau subbands. 


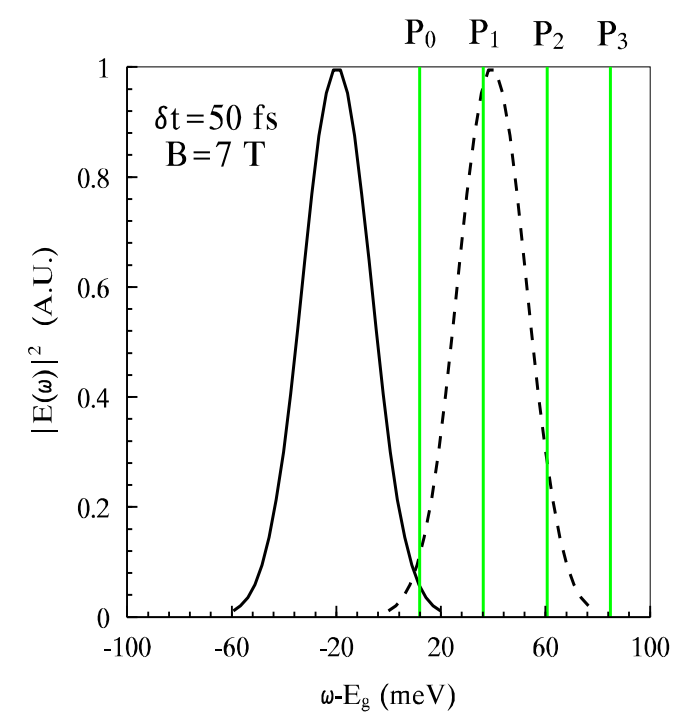

FIG. 2. The pulse intensity spectrum $|E(\omega)|^{2}$ for a 50 fs pulse tuned at $-20 \mathrm{meV}$ (solid curve) and $40 \mathrm{meV}$ (dashed curve), together with the unrenormalized band edges (with the label $P_{n}$ ) for the optical transitions between the Landau subbands $n(=0,1,2,3)$ plotted as solid lines for $B=7 \mathrm{~T}$. 


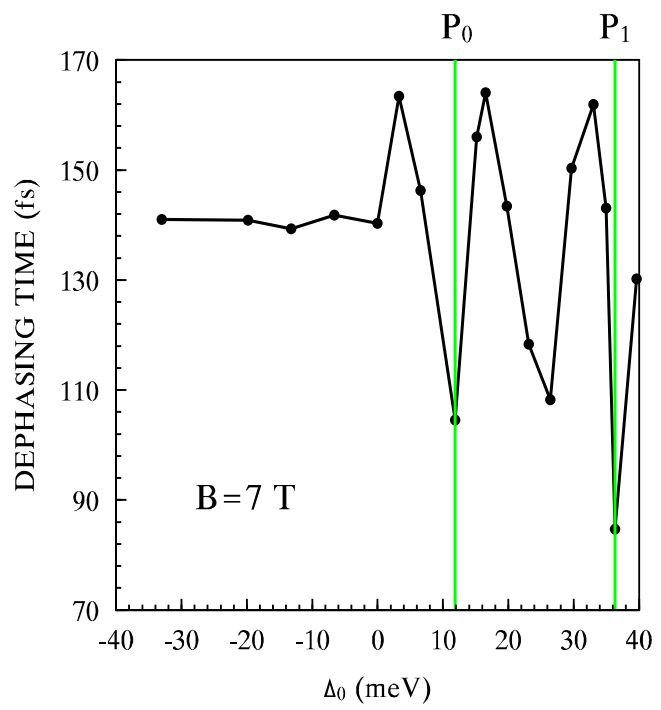

FIG. 3. Dephasing time as a function of detuning $\Delta_{0}$ for $B=7 \mathrm{~T}$. The solid lines are the unrenormalized band edges for the optical transition between the lowest two Landau subbands $n(=0$ and 1$)$. 